\title{
Research on Life Prediction Method for Gyroscope Based on PACE
}

\author{
Haodong Ma \\ Beijing Aerospace Measurement \& Control Corp. \\ Beijing Key Laboratory of High-Speed Transport Intelligent \\ Diagnostics and Health Management \\ Beijing, China \\ mahaodong@126.com
}

\section{Kai Luo}

Beijing Aerospace Measurement \& Control Corp. Beijing Key Laboratory of High-Speed Transport Intelligent Diagnostics and Health Management Beijing, China sam4321@163.com

\begin{abstract}
Gyroscopes are widely applied in weapon system and other high precision system. During the using course, the reliability and performance of gyroscope are influenced by environment and using condition. The performance of gyroscope degenerates periodically, and the remaining useful life (RUL) is payed close attention by engineers in order to monitor the whole systems steadily and safely during life-cycle. Traditionally, researchers used the reliability test and statistic method to estimate RUL. But it's difficult to get enough samples in the test because the gyroscope cost much. In this paper, we mainly discussed the life prediction of gyroscopes, designed the accelerated life testing(ALT), and used Path Classification and Estimation(PACE) to predict the RUL of gyroscopes. In addition, we analyzed the theory of life prediction for gyroscopes, mainly about the relationship of gyroscope's fault, nature element and useful life. Then the method is researched based on PACE method. At last, the prediction algorithms is illustrated by the practical accelerate life test data. The methods discussed in this paper was applied in a practical equipment maintenance support project, which can provide technical support for the product life prolong research work in the future.
\end{abstract}

Keywords-component; gyroscopes; remaining useful life; accelerated life testing; path classification and estimation

\section{INTRODUCTION}

As known, the gyroscope is the key component of navigation system, which calculate the angular velocity of the moving system as plane, ship, et. The precision of gyroscope decides the whole system navigation accuracy. In our research, it is required to calculate the gyroscope's RUL of a strapdown inertial navigation system(SINS). Because the gyroscope of SINS is fixed directly to the carrier, so the type of installation is not ideal[2]. The sensitive components' parameters are always shifting with the influence of environment and using conditions. This situation leads the stability of gyroscope to be unstable, and makes the whole system can't meet the mission

\author{
Rui Li \\ Beijing Aerospace Measurement \& Control Corp. \\ Beijing Key Laboratory of High-Speed Transport Intelligent \\ Diagnostics and Health Management \\ Beijing, China \\ 451198275@qq.com \\ Hongzheng Fang \\ Beijing Aerospace Measurement \& Control Corp. \\ Beijing Key Laboratory of High-Speed Transport Intelligent \\ Diagnostics and Health Management \\ Beijing, China \\ hongzhengf@163.com
}

requirements. With the influence grows further, the accuracy of gyroscope will grows unrecoverable, and the RUL comes to the end. Previous studies focus on the stability test and statistic calculate method. In our research, we has researched on more methods besides the traditional method, because we do not have enough test samples, and the constant stress mode of ALT ${ }^{[3]}$ will cost much time and price. This paper mainly discusses the three aspects: the influence from environment to the gyroscope's RUL, the improved test mode of ATL, and the PACE life prediction methods to RUL.

\section{FMEA OF GYROSCOPE}

The gyroscope of SINS is a typical mechanical and electrical equipment, which is composed of motor, circuits, sensors and some non-metallic materials. The gyroscope work on rate integrating principle, which calculates the angular deviation of the system. Fig. 1 shows us a typical gyroscope picture which can be got on Internet.

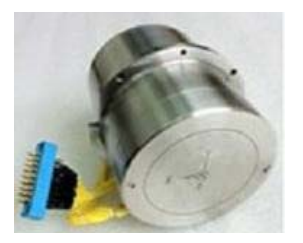

Figure 1. Rate integrating gyroscope

During the using time, the motor and sensor are always stable and not easy to fail. The main failure parts of gyroscope include electronic components such as capacitances and the glue which connects different components of gyroscope. As described above, the practical influence factors include the environments and the using condition, such as temperature, vibration, and power using time. The FMEA (Fault Modes and 
Effects Analysis) table of a type of gyroscope is shown by Table I.

TABLE I. THE MAIN FMEA OF GYROSCOPE

\begin{tabular}{|l|l|l|}
\hline \multicolumn{1}{|c|}{ Failure mode } & \multicolumn{1}{c|}{ Failure mechanism } & \multicolumn{1}{c|}{ Factor } \\
\hline axis coefficient drift & $\begin{array}{l}\text { Glue Aging, adhension } \\
\text { failure }\end{array}$ & Temperature \\
\hline no output & $\begin{array}{l}\text { Glue Aging, adhension } \\
\text { failure }\end{array}$ & Temperature \\
\hline output saturation & $\begin{array}{l}\text { Glue Aging, adhension } \\
\text { failure }\end{array}$ & Temperature \\
\hline Capacitance degradation & Leakage current increases & Temperature \\
\hline
\end{tabular}

From Table I, we can find that the gyroscope failure mode mainly occurred in glue and circuits, and the main factor is temperature. By further analysis, it can be found that sealing failure depends on temperature. Therefore we can ignore the impacts from humidity and vibration.

\section{RESEARCH PROCESS OF LIFE PREDICTION}

Because the gyroscope is a complex component and have independent functional, so it is not possible to research the glue and capacitances individually which can't analysis the interaction in gyroscope. At the same time, the gyroscope that we research on is a new product, so there are not enough normal test data which is used to build the life prediction modal So we need to obtain the test data by ALT in order to calculate the RUL of the gyroscope. We firstly finished the FMEA of gyroscope, and then implemented the ALT. From the ALT, we got test data, and extrpolated the data under high press to the normal press. At last we set up the PACE modal, and calculated the RUL of gyroscope. We will discuss the research by detail in the flowing sections.

\section{ALT OF GYROSCOPE’S}

\section{A. Design for gyroscope's ALT}

There are tree modes of ALT, which are constant stress, stepping stress and progressive stress that each mode has different features which is shown below.

TABLE II. DIFFERENT ModE'S FEATURES OF Alt

\begin{tabular}{|l|c|c|c|c|}
\hline \multicolumn{1}{|c|}{ Test mode } & Complexity & Samples & Economy & Time \\
\hline constant stress & Low & Many & High & High \\
\hline stepping stress & Medium & A few & Medium & Medium \\
\hline progressive stree & High & A few & Low & Low \\
\hline
\end{tabular}

The table above shows that the constant stress has high price, and the progressive stress has high complexity, so we ignored the two ALT modes and selected the stepping stress mode. But there were also some problems for the stepping stress mode, such as so much cost about time and money, and the product always do not failure under low stress which brings about the high-risk. Through extensive technical research, we find an improved method ${ }^{[3]}$ (Stepping down stress mode) which start the test from the high level while the traditional mode starts test from the low stress.

Before the testing, we have used two gyroscopes to do preparation testing in order to get the high limit stress level. The accurate stress level can reduce the testing cost and reduce the non-normal failure probability. We used one product to start the preparation testing, and recorded the performance and time under the $80^{\circ} \mathrm{C}$ until it failed in a short time. If the gyroscope worked well for 2 cycles, the testing went to the next temperature level. The last temperature minus $20^{\circ} \mathrm{C}$ is used to be 1 st stress level. The other product was used to test in 1 st stress level again to judge whether it worked normally or not. At last, we confirmed that $100^{\circ} \mathrm{C}$ to be the high stress level, and set the $40^{\circ} \mathrm{C}$ to be the low stress level.

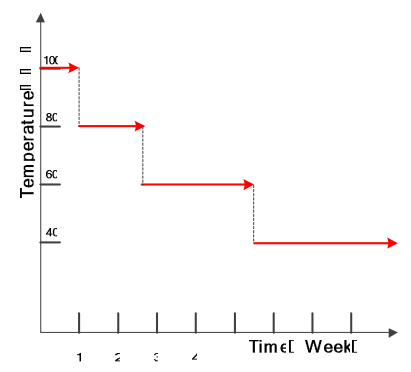

Figure 2. Test profile of the ALT

In the testing, we used 5 gyroscopes which are set in one group. If one product failed, we would stop the test, and went to the next stress. During the testing, the gyroscopes can't be tested online, so we have tested the gyroscopes after the heating process. Test data items ${ }^{[4]}$ in the table below.

TABLE III. GYroscope TEST ITEMS.

\begin{tabular}{|c|c|c|c|c|c|c|c|c|}
\hline \multirow[t]{2}{*}{ Num } & \multicolumn{3}{|c|}{ Axial } & \multicolumn{3}{|c|}{$\begin{array}{c}\text { acceleration } \\
\text { component } \\
\text { along the axis }\end{array}$} & \multicolumn{2}{|c|}{$\begin{array}{c}\text { acceleration } \\
\text { component of the } \\
\text { Earth's rotation } \\
\end{array}$} \\
\hline & $\mathbf{X}$ & $\mathbf{Y}$ & $\mathbf{Z}$ & $\mathbf{X}$ & $\mathbf{Y}$ & $\mathbf{Z}$ & $\mathbf{X}$ & $\mathbf{Y}$ \\
\hline 1 & $\mathrm{~N}$ & W & $\mathrm{U}$ & 0 & 0 & 1 & $\omega_{e} \cos \varphi$ & 0 \\
\hline 2 & W & S & $\mathrm{U}$ & 0 & 0 & 1 & 0 & $-\omega_{e} \cos \varphi$ \\
\hline 3 & $\mathrm{~N}$ & $\mathrm{E}$ & $\mathrm{U}$ & 0 & 0 & 1 & $-\omega_{e} \cos \varphi$ & 0 \\
\hline 4 & $\mathrm{E}$ & $\mathrm{N}$ & $\mathrm{U}$ & 0 & 0 & 1 & 0 & $\omega_{e} \cos \varphi$ \\
\hline 5 & E & $\mathrm{D}$ & $\mathrm{N}$ & 0 & -1 & 0 & 0 & $-\omega_{e} \sin \varphi$ \\
\hline 6 & $\mathrm{U}$ & $\mathrm{E}$ & $\mathrm{N}$ & 1 & 0 & 0 & $\omega_{e} \sin \varphi$ & 0 \\
\hline 7 & $\mathrm{~W}$ & $\mathrm{U}$ & $\mathrm{N}$ & 0 & 1 & 0 & 0 & $\omega_{e} \sin \varphi$ \\
\hline 8 & $\mathrm{D}$ & $\mathrm{W}$ & $\mathrm{N}$ & -1 & 0 & 0 & $-\omega_{e} \sin \varphi$ & 0 \\
\hline \multicolumn{9}{|c|}{$\begin{array}{l}\text { N-North, W-West, S-South, E-East, U-Up, D-Down, } \\
\omega_{e}-\text { Earth's rotation angular velocity, } \\
\varphi_{-} \text {Local latitude angle. }\end{array}$} \\
\hline
\end{tabular}

\section{B. Data Extrapolation of ALT}

We have tested the ALT data after every heating process, and extrapolated data from high stress to low stress. First, 
assume test stress as $S_{K}>S_{K-1}, \ldots,>S_{1}, \mathrm{n}$ is test samples number and test times under different stresses are $\tau_{K}>\tau_{K-1}, \ldots,>\tau_{1}$. Gyroscope's failure time under $S_{i}$ stress meets (1).

$$
t_{i 1} \leq t_{i 2} \leq \ldots \leq t_{i r_{i}}, i=1,2, \ldots, k
$$

As known, RUL or performance of gyroscope obeys the Weibull distribution, the probability density function is expressed as follow.

$$
f_{i}(t)=\frac{m_{i}}{\eta_{i}}\left(\frac{t}{\eta_{i}}\right)^{m_{i}-1} e^{\left\{-\left(\frac{t}{\eta_{i}}\right)^{m_{i}-1}\right\}}, i=0,1, \ldots, k
$$

Then, we will get the follow accelerate equation by Arrehennius equation ${ }^{[5]}$ :

$$
\ln h_{i}=a+b j\left(S_{i}\right), i=0,1, \ldots, k
$$

Based on Nelson cumulative exposure model, the remaining life depends on cumulative exposure and the stress, instead of the cumulative mode.

According to (3), we can get the samples under the stress $S_{1}$ which has the same probability of failure with the samples under the stress $\mathrm{S}_{j}$, as below.

$$
1-\exp \left\{-{\frac{t_{i}}{h_{i}}}^{m_{i}}\right\}=1-\exp \left\{-{\frac{t_{j}}{h_{j}}}^{m_{j}}\right\}
$$

For (4), we can use parameter estimation method to calculate the parameter $\mathrm{m}$ and $\eta$, first set as below.

$$
\begin{aligned}
& \mathrm{EX}^{\mathrm{a}}=\frac{1}{\mathrm{n}} \sum_{\mathrm{i}=1}^{\mathrm{n}} \mathrm{X}_{\mathrm{i}}^{\mathrm{a}}, \mathrm{EX}^{-\mathrm{a}}=\frac{1}{\mathrm{n}} \sum_{\mathrm{i}=1}^{\mathrm{n}} \mathrm{X}_{\mathrm{i}}^{-\mathrm{a}} \text {, then we can get: } \\
& \hat{\mathrm{m}}=\frac{\mathrm{a} \mathrm{A}(2 \mathrm{a})}{\mathrm{A}(\mathrm{a}) \sqrt{\mathrm{A}^{2}(2 \mathrm{a})-\mathrm{A}^{2}(\mathrm{a})}} \\
& \hat{\eta}=\frac{\mathrm{B}(\mathrm{a})}{\Gamma(1+\mathrm{a} / \mathrm{m})}
\end{aligned}
$$

Where

$$
\mathrm{A}(\mathrm{a})=\frac{1}{\mathrm{n}} \sum_{\mathrm{i}=1}^{\mathrm{n}} \mathrm{X}_{\mathrm{i}}^{\mathrm{a}} \frac{1}{\mathrm{n}} \sum_{\mathrm{i}=1}^{\mathrm{n}} \mathrm{X}_{\mathrm{i}}^{-\mathrm{a}}, \quad \mathrm{B}(\mathrm{a})=\frac{1}{\mathrm{n}} \sum_{\mathrm{i}=1}^{\mathrm{n}} \mathrm{X}_{\mathrm{i}}^{\mathrm{a}}
$$

We set the test data of samples as $t_{j}\left(\eta_{i}\right)$, and then can get the flowing equitation:

$$
U_{j}\left(m_{i}, \eta_{i}\right)=\sum_{k=1}^{j} t_{k}^{m_{i}}\left(\eta_{i}\right)+(n-j) t_{j}^{m_{i}}\left(\eta_{i}\right),\left(j=1,2, \ldots, n_{i}\right)
$$

\section{Life Prediction Method of PACE}

PACE(Path Classification and Estimation) ${ }^{[1]}$ is a reliability of statistical methods which is fit to solute the small samples question. The method derived from GPM(General Path Model). PACE uses the performance of products and the reliability of statistical to build the performance degradation path. By comparing the path of target system with other paths, we can get the result of the RUL of the target system. Generally, performance degradation path is not irregular that we can use regression analysis algorithm to smooth the curve of the performance degradation path.

There are two main processes to build up the degradation path.

- $\quad$ First, compare the target path with the other paths, and calculate the similarity of the target path with the other paths.

- Second, use the similarity to estimate RUL of the target system.

Set the degenerate amount when time is $t^{*}$ as $f(t, \Theta)$.

$$
f\left(t^{*}, \Theta\right)=\left[\begin{array}{l}
f_{1}\left(t^{*}, \theta_{1}\right) \\
f_{2}\left(t^{*}, \theta_{2}\right) \\
f_{3}\left(t^{*}, \theta_{3}\right) \\
f_{4}\left(t^{*}, \theta_{4}\right)
\end{array}\right]
$$

Where 1,2,3,4 represent different degradation path. Then RUL can be expressed as follow.

$$
L\left(t^{*}\right)=T-t^{*}=\left[\begin{array}{c}
T_{1}-t^{*} \\
T_{2}-t^{*} \\
T_{3}-t^{*} \\
T_{4}-t^{*}
\end{array}\right]
$$

Kernel regression method can be used to calculate the membership for every degradation path. The memberships of all the degradation paths can be made up to a membership vector as follow.

$$
\mu_{U}\left[u\left(t^{*}\right)\right]=\left[\begin{array}{l}
\mu_{U_{1}}\left[u\left(t^{*}\right)\right] \\
\mu_{U_{2}}\left[u\left(t^{*}\right)\right] \\
\mu_{U_{3}}\left[u\left(t^{*}\right)\right] \\
\mu_{U_{4}}\left[u\left(t^{*}\right)\right]
\end{array}\right]
$$

For the kernel regression method, we need tree main steps to calculate the unknown parameters ${ }^{[6]}$.

- Calculate distance between the target path and other paths of samples.

- Calculate the similarity of the paths using the Gaussian kernel function, et.Gaussian kernel function:

$$
w_{i}=K_{h}\left(d_{i}\right)=\frac{1}{\sqrt{2 \pi h^{2}}} e^{-d_{i}^{2} / 2 h^{2}}
$$


- Use the similarity plus the weights, and get the RUL of the target system, as follow.

$$
\hat{y}(x)=\frac{1}{a} \sum_{i=1}^{n}\left(w_{i} Y_{i}\right)
$$

\section{LifE PREDiction ANALysis of Gyroscope}

\section{A. Traditional Statistical Life Prediction Analysis}

In the gyroscopes' testing, we selected 5 gyroscopes as the test samples, and tree samples failed under $100^{\circ} \mathrm{C}, 80^{\circ} \mathrm{C}, 60^{\circ} \mathrm{C}$. Under $40^{\circ} \mathrm{C}$, there were no samples failed, so we selected the pre-design time to stop the testing. After the testing, we extrapolated the performance and time under the $100^{\circ} \mathrm{C}, 80^{\circ} \mathrm{C}$, $60^{\circ} \mathrm{C}, 40{ }^{\circ} \mathrm{C}$ to the normal temperature $20^{\circ} \mathrm{C}$.

Beside PACE, we first used the traditional reliability statistics method to get the character life of gyroscope. Fig. 4 is the fitting result on Weibull model.

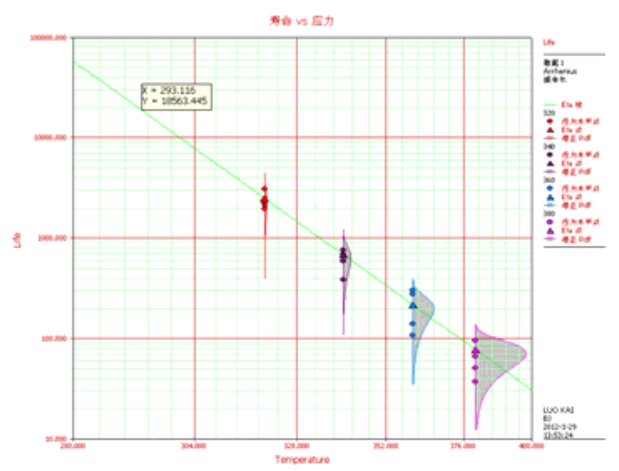

Figure 3. Life-Stress

\section{B. Life Prediction Based on PACE}

In PACE, we use the $|\mathrm{D}(\mathrm{Y}) \mathrm{y}|$ of gyroscopes as degradation of performance to build up the degradation paths. Because the $40^{\circ} \mathrm{C}$ test data have no failure time, so we increased the failure threshold which gyroscopes have arrived the failure threshold under every stress. The Initial performance of gyroscopes is shown as below.

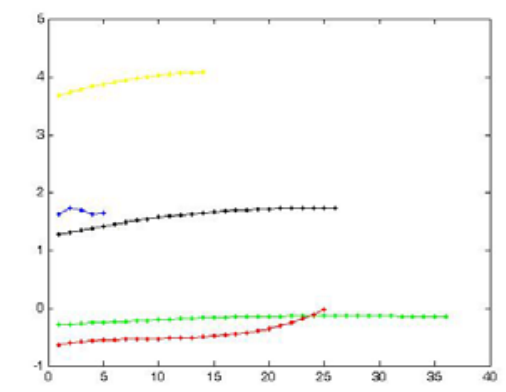

Figure 4. five gyroscopes' performance curves

For each gyroscope, we used the extrapolated performance as the target system to construct the kernel function and calculate the weights. One of the gyroscopes' degradation path was built up as follow.

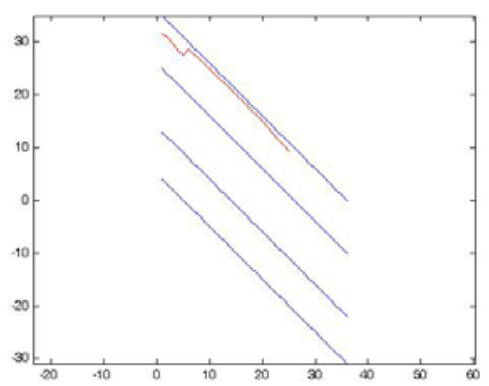

Figure 5. One gyroscope's degradation path

As Fig.5 shown, we can see that the pink curve is the target gyroscope's degradation path, and the blues are the other four gyroscope's degradation paths. At last, we got that the RUL of the five gyroscopes are respectively 20.3813, 64.6029, 35.4745 and 43.1495 months.

\section{CONCLUSION}

Based on our research work, it is concluded that the traditional statistics methods can't solute the small samples products and give RUL of every product's. PACE can solve the problems above. More precisely, the traditional statistics distribution is just life evaluation method. At the same time, PACE life prediction method is not verified in this paper for lacking of the practical failure gyroscopes' performance and failure time. In the further, we will use the practical gyroscopes' historical failure data under normal stress to build the degradation paths and finish the verification work. With verified result, it is needed to improve PACE method in order to meet the engineering application needs.

\section{REFERENCES}

[1] MA Jiming, ZHAN Xiaoyan, "Performance Reliability Analysis of a Piston Pump Affected by Random Degradation," Journal of Mechanical Engineering, vol.46, pp. 612-618, July 2010.

[2] D. H. Titterton, J. L. Weston, Strapdown Inertial Navigation Technology, 2nd ed., The Institution of Electrical Engineers, 2005, pp.2,152-153.

[3] Zhang Chunhua, "Theory and Method of Step down stress Accelerated Life Testing," Ihistitute of Meehatronical Engineering \& Automation National University of Defense Teehnology, Changsha, China, April 2002.

[4] "QJ 1079A-2004:Major precision parameters and testing methods for gyroscopes", September 2004, p.11.

[5] S. S. Mao, L. G. Wang, Accelerated Life Test, Science Press, 2000, pp.588.

[6] WANG Ping-bo, CAI Zhi-ming, LIU Wang-suo, "Dynamic cluster algorithm for Gaussian mixture parameter estimation", Technical Acoustics, vol.26, pp741-746, August 2007.

[7] Kai Luo, Liming Han, Hongzheng Fang, Shumin Hou, "Research on Storage Life Prediction Method for Strapdown Inertial Navigation System", Prognostics \& System Health Management Conference, vol4, pp. 516-521, July 2012. 